\title{
Combinations in multimodality treatments and clinical outcomes during cancer (Review)
}

\author{
XIAO-YING ZHANG ${ }^{1}$ and PEI-YING ZHANG ${ }^{2}$ \\ ${ }^{1}$ Nanjing University of Chinese Medicine, Information Institute, Nanjing; ${ }^{2}$ Department of Cardiology, Xuzhou Central \\ Hospital, The Affiliated Xuzhou Hospital of Medical College of Southeast University, Xuzhou, Jiangsu 221009, P.R. China
}

Received April 27, 2016; Accepted October 3, 2016

DOI: $10.3892 / \mathrm{ol} .2016 .5242$

\begin{abstract}
Combination approach could be easily considered as the future of therapeutics in all pathological states including cancer. Scientists are trying different combinations in order to determine synergism among different therapeutics which ultimately helps in the improved and more efficient management of the affected patients. Combination of multi-chemotherapeutic agents, or multi-drug therapy, may be the most commonly used strategy for cancer treatment. Monotherapy causes drug resistance and loses its response in patients after several cycles of treatment. While combining different anticancer drugs together for cancer treatment, as in the case of the cocktail therapy for HIV, not only overcomes the drug resistance but also leads to a synergistic effect, therefore showing prolonged survival for patients. The present review article is focused on different combinations in use for better efficiency of therapeutics against cancer. We searched the electronic database PubMed for pre-clinical as well as clinical controlled trials reporting diagnostic as well as therapeutic advances of various combinations in cancer. It was observed clearly that combination approach is better in various aspects including increase in efficacy, specificity and decline in the unwanted side effects.

Contents

1. Introduction
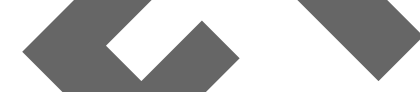

2. Combination of hyperthermia therapy

3. Combination of anti-angiogenesis therapy

4. Combination of photodynamic therapy

5. Combination of gene therapy

6. Conclusion
\end{abstract}

Correspondence to: Dr Pei-Ying Zhang, Department of Cardiology, Xuzhou Central Hospital, The Affiliated Xuzhou Hospital of Medical College of Southeast University, 199 South Jiefang Road, Xuzhou, Jiangsu 221009, P.R. China

E-mail: xiaoyingzhang08@163.com

Key words: combinations, cancer therapeutics, multimodal treatments

\section{Introduction}

Cancer immunotherapy is an evolving avenue in cancer therapeutics (1). It involves antigen-presenting cells (dendritic cells) that become mature after presentation of antigens and eventually migrate towards lymph nodes. This process further induces $\mathrm{T}$ cell and natural killer (NK) cell responses in lymphoid organs.Finally, such T cells (also B cells and NK cells) exit the lymph node, followed by entry into the tumor bed, stimulating immune response and resulting in antitumor effect (2). The immunotherapeutic agents included melanoma-differentiation antigens such as MART-1, gp100, tyrosinase or TRP-2; cancer-testes antigens such as NY-ESO-1 or MAGE-12 (3); monoclonal antibodies targeting cancer-associated proteins of Her2/neu, epidermal growth factor receptor, vascular endothelial growth factor, CD20, CD52 or CD33. Immunostimulatory monoclonal antibodies are also used, including antagonist antibodies such as anti-cytotoxic $\mathrm{T}$ lymphocyte antigen-4 (CTLA-4), anti-programmed death-1 (PD-1), anti-KIR and anti-transforming growth factor- $\beta$; and agonist antibodies targeting CD40, CD137, CD134 and glucocorticoid-induced TNF receptor (4). Additionally, cytokines such as interleukin (IL)-2, granulocyte-macrophage colony-stimulating factor and interferon- $\alpha$ are also delivered for immunotherapy (5).

Cancer vaccines used in immunotherapy are often not sufficient enough for tumor response. The rationale behind combination immunotherapy is various. One strategy is multi-immunotherapy which combines different agents to target one or more stages of the immune response, stimulating the T-cell response and overcoming the immunosuppression simultaneously. For example, it is found that $\mathrm{T}$ cells express multiple inhibitory receptors (6). Combining immune stimulator such as IL-15 with multiple blockades for inhibitory receptors, such as CTLA-4, PD1 and PD ligand-1, have been reported to enhance immune response (7). The trial showed a better efficacy for multimodality treatment among chemo-, radio- and immunotherapy (8).

\section{Combination of hyperthermia therapy}

Hyperthermia therapy, chemotherapy and/or radiotherapy are often combined together for cancer therapy. Hyperthermia is used as an efficient adjuvant treatment with radiotherapy and/or chemotherapy because it causes tumor reoxygenation. 
When tumors are heated up between 39 and $43^{\circ} \mathrm{C}$, improvement in oxygenation is emerged (9). The resultant reoxygenation may last for $24 \mathrm{~h}$ after heating (10). It has been demonstrated that hypoxia plays a role in chemo- and radioresistance. The improvement of tumor oxygenation might increase the possibility of a positive response to radiation therapy (11). In addition, combination with hyperthermia have been reported to make tumors more sensitive towards chemotherapy as well as radiotherapy (12).

The multimodality treatment of intracavitary hyperthermia and chemoradiotherapy provided an effective and safe therapeutic modality (13). The above combination has also applied in a clinical trial involving triple-modality treatment combined full-dose radiotherapy, chemotherapy and locoregional hyperthermia therapy during advanced cervical carcinoma. After a median follow-up of 538 days, $74 \%$ of patients survived without signs of recurrence, and the overall survival rate was $84 \%$. Long-term survival data showed that the 5-year recurrence-free survival rate was $57.5 \%$ and the 5 -year overall survival was $66.1 \%(14,15)$. In another combination treatment of paclitaxel, liposomal doxorubicin and local breast hyperthermia, survival of $75 \%$ has been reported to be increased by five years (16).

Further, gemcitabine and cisplatin with regional hyperthermia also had better efficacy in advanced pancreatic cancer patients (17). The regional hyperthermia in combination with platinum-based chemotherapy was also tested on childrenand adolescents with recurrent or refractory germ cell tumors. It revealed that 7 of 10 patients had objective tumor response (18)

Apart from the increased chemo- or radio-sensitivity induced by heat, hyperthermia caused denaturation and aggregation of proteins to influence DNA repair and cell cycle regulation, changed the vascularity for enhanced blood flow and drug delivery, up-regulated the expression of genes to produce the family of heat shock proteins (HSPs) (19). The overexpression of HSPs turned out to be related to thernoresistance (20). Suppression the gene expression of HSPs is another kind of multimodality reatment which stimulated the T-cell immune response (21). Additionally, gold nanoparticles could be used as photothermal agents in combination with immnotherapy (22). Carbon nanotubes are agents for hyperthermia based on radiofrequency ablation or absorbance of near-infrared radiation $(23)$

\section{Combination of anti-angiogenesis therapy}

Anti-angiogenesis therapy inhibits growth of cancer by blocking the formation of new blood vessels (24). Angiogenesis is often involved in tissue regeneration and in chronic inflammatory conditions. Cancer will benefit from the new blood vessels for oxygen and nutrients, which allow cancer cells to multiply, invade nearby tissue, and metastasis (25). Angiogenesis inhibitor is a type of chemical, which interferes with the signals related to formation of new blood vessels. There are two kinds of inhibitors: Direct angiogenesis inhibitor and indirect one. Direct angiogenesis inhibitor restrains response of vascular endothelial cells to pro-angiogenic proteins; whereas indirect inhibitor blocks the synthesis of angiogenic proteins by tumor cells, reduces the activity of the proteins or blocks the expression of receptors on endothelial cells (26).
Anti-angiogenesis therapy is often combined with chemotherapy, radiotherapy or immunotherapy for additive or synergistic effect (27). By 'normalizing' tumor vessels through anti-angiogenesis therapy, it leads to decreased vascular leakage, lower intratumoral-tissue pressure and increased delivery of therapeutic agents. Due to the decreased intratumoral pressure, anti-angiogenesis therapy also increases oxygenation supply in tumor and leads to sensitivity of a tumor for ionizing radiation. The improvement in blood flow and oxygenation also leads to higher level of glucose and oxygen as well as amino acids, which will help $\mathrm{T}$ cells to keep activity in immunotherapy. Combining anti-angiogenesis therapy with immunotherapy enhances dendritic-cell function, decreases the number of myeloid-derive stem cells and regulatory $\mathrm{T}$ cells, and increase lymphocyte infiltration. In a recent study study, dendritic cell-based autologous whole tumor vaccination was utilized together with anti-angiogenesis therapy, followed by transfer of autologous vaccine-primed CD3/CD28-co-simulatied lymphocytes for recurrent ovarian cancer patients (28). In another trial, anti-angiogenesis therapy was combined with hormonal therapy on patients with hormone receptor positive breast cancer, who received first-line bevacizumab and taxane-including regimen (29).

\section{Combination of photodynamic therapy}

Photodynamic therapy (PDT) utilizes photosensitizing agent and a particular type of light to cure cancer. When the photosensitizing agent is accumulated in a tumor, a laser or other source of light is delivered to the areas at the same time. The photosensitizer in tumor gets activated from a ground to an excited state and release energy by returning to its ground state. The energy is then transferred to oxygen and results in generation of reactive oxygen species (ROS), which causes cellular toxicity (30). The most commonly used photosensitizer is paophyrin. Other classes of photosensitizers include chlorin, bacteriochlorin, phthalocyanine, phenothiazinium compound, porphycene, hypericin, chlorophyll derivative, texaphyrin, antracen, purpurin and hypocrellin. Among them, 5-aminolevulinic acid, methyl 5-aminolevulinate, methyl-tetrahydroxyphenyl chlorine and haematoporphyrin derivative are approved for clinical use (31).

Apart from the direct killing of cancer cells by generation of ROS, PDT also damages tumor-associated vasculature, leading to tumor hypoxia/anoxia and nutrient deficiency. It also activates immune response against tumor cells and decreases immunosuppressive effect (32). Currently, there are many clinical trials using PDT alone (33). However, most of the photodynamic-related multimodality treatments are still studied in animal models (34). There is one phase I clinical trial in which PDT was combined with surgery. The trial demonstrated the feasibility of combination with surgery and PDT (35).

\section{Combination of gene therapy}

Gene therapy is a novel treatment that involves the introduction of genetic material (DNA or RNA) into a person's cells to fight disease. Viral vectors have been used to deliver genes to tumor cells (36). Gene therapy could be a direct attack on 
tumor cells. Delivery of tumor-suppressor genes to cancer cells and successful expression of them, or delivery of antisense oligonucleotides or rebozymes to block oncogene expression, leads to cell death or growth inhibition. Additionally, delivery of suicide genes, which are enzyme-encoding genes for metabolizing a harmless prodrug, could activate prodrugs for cytotoxicity and lead to a bystander effect. Except for the direct attack on tumor cells, gene therapy also stimulates one's immune response, target to prevent angiogenesis, or overcome the cell resistance and make cancer cells more sensitive to other therapies, such as chemotherapy and radiotherapy (37). Theoretically, gene therapy has the ability to combine with all the above therapies including chemotherapy, immunotherapy, hyperthermia therapy, anti-angiogenesis therapy and PDT by targeting certain pathways of cancer cells and promote the efficacy of other therapies. Recent cutting edge research is concerned with RNA interference (RNAi) therapy involving microRNA and small interfering RNA (38). It shows the potential of anticancer effect through inhibiting over-expressed oncogenes, blocking cell division and promoting apoptosis. It also suppresses therapeutic resistance to enhance other therapies (39).

Currently, there are many clinical trials for gene therapy alone without combination with other therapies (40). Many combination gene therapies are still studied at preclinical level. RNAi gene therapy has come to clinical trials but not combined with other therapy modalities. One example of combination gene therapy was conducted on prostate cancer patients with herpes simplex virus-thymidine kinase (41).

\section{Conclusion}

It can be concluded from the above approach is crucial in enhancing the peutics in variable therapeutic app

\section{References}
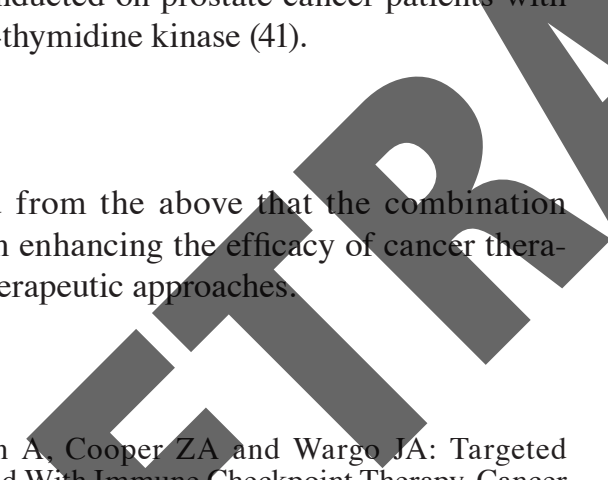

\section{7.}

1. Prieto PA, Reuben A, Cooper ZA and Wargo JA: Targeted Therapies Combined With Immune Checkpoint Therapy. Cancer J 22: 138-146, 2016

2. Zhang Z, Guo Y and Feng SS: Nanolmmunotherapy: Application of nanotechnology for sustained and targeted delivery of antigens to dendritic cells. Nanomedicine (Lond) 7: 1-4, 2012.

3. Rosenberg SA, Yang JC and Restifo NP: Cancer immunotherapy: Moving beyond current vaccines. Nat Med 10: 909-915, 2004.

4. Melero I, Grimaldi AM, Perez-Gracia JL and Ascierto PA: Clinical development of immunostimulatory monoclonal antibodies and opportunities for combination. Clin Cancer Res 19: 997-1008, 2013.

5. Mellman I, Coukos G and Dranoff G: Cancer immunotherapy comes of age. Nature 480: 480-489, 2011.

6. Blackburn SD, Shin H, Haining WN, Zou T, Workman CJ, Polley A, Betts MR, Freeman GJ, Vignali DA and Wherry EJ: Coregulation of $\mathrm{CD} 8^{+} \mathrm{T}$ cell exhaustion by multiple inhibitory receptors during chronic viral infection. Nat Immunol 10: 29-37, 2009.

7. Yu P, Steel JC, Zhang M, Morris JC and Waldmann TA: Simultaneous blockade of multiple immune system inhibitory checkpoints enhances antitumor activity mediated by interleukin-15 in a murine metastatic colon carcinoma model. Clin Cancer Res 16: 6019-6028, 2010.

8. Ramos-Suzarte M,Lorenzo-Luaces P, Lazo NG, et al: Treatment of malignant, non-resectable, epithelial origin esophageal tumours with the humanized anti-epidermal growth factor antibody nimotuzumab combined with radiation therapy and chemotherapy. Cancer Biol Ther 13: 600-605, 2012.
7. Tschoep-Lechner KE, Milani V, Berger F, Dieterle N, Abdel-Rahman S, Salat C and Issels RD: Gemcitabine and cisplatin combined with regional hyperthermia as second-line treatment in patients with gemcitabine-refractory advanced pancreatic cancer. Int J Hyperthermia 29: 8-16, 2013.

. Wessalowski R, Kruck H, Pape H, Kahn T, Willers R and Göbel U: Hyperthermia for the treatment of patients with malignant germ cell tumors: A phase I/II study in ten children and adolescents with recurrent or refractory tumors. Cancer 82: 793-800, 1998.

19. Issels RD: Hyperthermia adds to chemotherapy. Eur J Cancer 44: 2546-2554, 2008.

20. Lindquist S and Craig EA: The heat-shock proteins. Annu Rev Genet 22: 631-677, 1988.

21. Kumar CS and Mohammad F: Magnetic nanomaterials for hyperthermia-based therapy and controlled drug delivery. Adv Drug Deliv Rev 63: 789-808, 2011.

. Dong JM, Zhao SG, Huang GY and Liu Q: NADPH critically required for survival of undifferentiated human promyelocytic leukemia cell line HL-60. Free Radic Res 38: 629-637, 2004.

from the clinic to the laboratory: A hypothesis. Int J Hyperthermia 11: 315-322, 1995.

1. Vujaskovic Zand Song CW: Physiological mechanisms underlying
heat-induced radiosensitization. Int J Hyperthermia 20: 163-174, 2004.

Miraux $\mathrm{S}$, Thiaudière $\mathrm{E}$, Tan $\mathrm{S}$, Brisson $\mathrm{A}$, et al: Doxorubicin for MR imaging and magneto-chemotherapy. ACS Nano 5: 1122-1140, 2011.

Uzunoglu N, Papavasiliou C and Vlahos L: Chemoradiotherapy combined with intracavitary hyperthermia for anal cancer: Feasibility and long-term results from a phase II randomized Westermann AM, Jones EL, Schem BC, triple-modality treatment combining radiotherapy, chemotherapy, and hyperthermia for the treatment of patients with stage IIB, . Westermann A, Mella O, Van Der Zee J, Jones ELE, Van Der Velden J, Burger C, et al: Uong-term survival data of triple modality treatment of stage IIB-III-IVA cervical cancer with the combination of radiotherapy, chemotherapy and hyperVujaskovic Z, Kim DW, Jones E, Lan L, McCall L, Dewhirst MW, raciunescu O, StaufferP, Liotcheva V, Betof A and Blackwell K: phase I/II study of neoadjuvant liposomal doxorubicin, aclitaxel, and hyperthermia in locally advanced breast cancer.

. Boisselier E and Astruc D: Gold nanoparticles in nanomedicine: Preparations, imaging, diagnostics, therapies and toxicity. Chem Soc Rev 38: 1759-1782, 2009.

23. Day ES, Morton JG and West JL: Nanoparticles for thermal cancer therapy. J Biomech Eng 131: 074001, 2009.

24. Kerbel R and Folkman J: Clinical translation of angiogenesis inhibitors. Nat Rev Cancer 2: 727-739, 2002.

25. Folkman J: Angiogenesis: An organizing principle for drug discovery? Nat Rev Drug Discov 6: 273-286, 2007.

26. Folkman J: Angiogenesis. Annu Rev Med 57: 1-18, 2006.

27. Kamrava M, Bernstein MB, Camphausen K and Hodge JW: Combining radiation, immunotherapy, and antiangiogenesis agents in the management of cancer: The Three Musketeers or just another quixotic combination? Mol Biosyst 5: 1262-1270, 2009.

28. Kandalaft LE, Powell DJ Jr, Chiang CL, Tanyi J, Kim S, Bosch M, Montone K, Mick R, Levine BL, Torigian DA, et al: Autologous lysate-pulsed dendritic cell vaccination followed by adoptive transfer of vaccine-primed ex vivo co-stimulated T cells in recurrent ovarian cancer. OncoImmunology 2: e22664, 2013.

29. Fabi A, Russillo M, Ferretti G, Metro G, Nisticò C, Papaldo P, De Vita F, D'Auria G, Vidiri A, Giannarelli D and Cognetti F: Maintenance bevacizumab beyond first-line paclitaxel plus bevacizumab in patients with Her2-negative hormone receptor-positive metastatic breast cancer: Efficacy in combination with hormonal therapy. BMC Cancer 12: 482, 2012.

30. Dolmans DEJGJ, Fukumura D and Jain RK: Photodynamic therapy for cancer. Nat Rev Cancer 3: 380-387, 2003. 
31. Baldea I and Filip AG: Photodynamic therapy in melanoma - an update. J Physiol Pharmacol 63: 109-118, 2012.

32. Zuluaga MF and Lange N: Combination of photodynamic therapy with anti-cancer agents. Curr Med Chem 15: 1655-1673, 2008.

33. Brown SB, Brown EA and Walker I: The present and future role of photodynamic therapy in cancer treatment. Lancet Oncol 5: 497-508, 2004

34. Agostinis P, Berg K, Cengel KA, Foster TH, Girotti AW, Gollnick SO, Hahn SM, Hamblin MR, Juzeniene A, Kessel D, et al: Photodynamic therapy of cancer: An update. CA Cancer J Clin 61: 250-281, 2011.

35. Friedberg JS, Mick R, Stevenson J, Metz J, Zhu T, Buyske J, Sterman DH, Pass HI, Glatstein E and Hahn SM: A phase study of Foscan-mediated photodynamic therapy and surgery in patients with mesothelioma. Ann Thorac Surg 75: 952-959, 2003.
36. Thomas CE, Ehrhardt A and Kay MA: Progress and problems with the use of viral vectors for gene therapy. Nat Rev Genet 4 : 346-358, 2003.

37. McCormick F: Cancer gene therapy: Fringe or cutting edge? Nat Rev Cancer 1: 130-141, 2001.

38. DeVincenzo JP: The promise, pitfalls and progress of RNA-interference-based antiviral therapy for respiratory viruses. Antivir Ther 17 (1 Pt B): 213-225, 2012.

39. Izquierdo M: Short interfering RNAs as a tool for cancer gene therapy. Cancer Gene Ther 12: 217-227, 2005.

40. Ginn SL, Alexander IE, Edelstein ML, Abedi MR and Wixon J: Gene therapy clinical trials worldwide to 2012 - an update. J Gene Med 15: 65-77, 2013.

41. Davidson BL and McCray PB Jr: Current prospects for RNA interference-based therapies. Nat Rev Genet 12: 329-340, 2011.

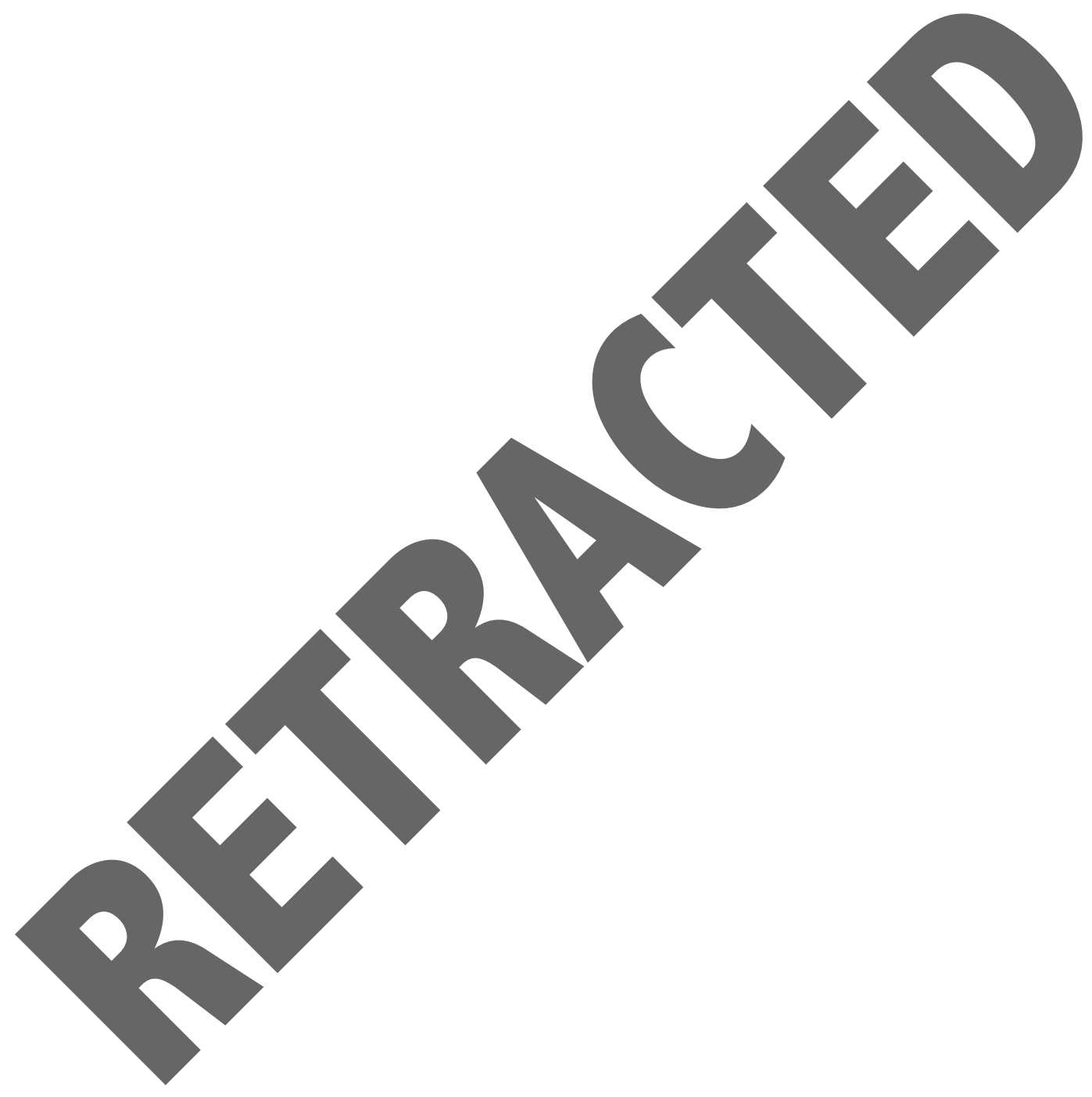

